\title{
Factors driving co-occurrence of Schistosoma mansoni and S. haematobium at the micro- geographical level
}

\author{
Lynn Meurs ${ }^{1 *}$, Moustapha Mbow ${ }^{2}$, Maria Yazdanbakhsh $^{3}$, Katja Polman ${ }^{1}$ \\ From Methods in Epidemiology Symposium \\ Leuven, Belgium. 17 September 2015
}

\section{Brief introduction}

Several studies have shown that the two major human Schistosoma species, Schistosoma mansoni and Schistosoma haematobium, co-exist in the same individuals at the micro-geographical level. However, the drivers of this co-occurrence are still largely unknown. Here, we studied whether coexposure and/or host immune responses might explain this phenomenon.

\section{Methods and materials}

A multidisciplinary study was conducted in two neighboring rural communities in northern Senegal, where $S$. mansoni and S. haematobium are co-endemic $(\mathrm{n}=857)$. Kato-Katz and urine filtration were used for microscopic detection of the respective Schistosoma species. Households were located using handheld differential global positioning system in the largest community $(n=599)$, and the Kulldorff's scan statistic was used to detect spatial clusters of infection. Schistosoma-specific cytokine responses (IL-10, IL-5, IFN- $\gamma$, TNF-a, and IL-2) were assessed in $72 \mathrm{~h}$ whole blood culture supernatants $(n=200)$, and analyzed by the multivariate technique nonmetric multidimensional scaling (nMDS).

\section{Results}

Classical epidemiological analyses showed that the two parasites were more likely to co-occur in the same individuals $(\mathrm{p}<0.001)$. Moreover, co-infected subjects had significantly higher infection levels than their monoinfected counterparts $(\mathrm{p}<0.001$; adjusted for age and gender). In contrast, micro-geographical analyses revealed a very focal spatial distribution with $S$. mansoni clustering in one $(\mathrm{p}=0.002)$ and S. haematobium infections in another section $(\mathrm{p}=0.023)$ of the community. nMDS analysis of the cytokine data indicated that the characteristic modified Th2 response was most pronounced in co-infected subjects.

\section{Conclusions}

The divergent geographical distribution of S. mansoni and S. haematobium in this community could not explain why the two infections cluster in the same individuals. This implies that co-infection is not driven by coexposure, but by within-host interactions. Cytokine profiles suggested that co-occurrence of the two species may be due to host immunological factors and/or parasite-induced immunomodulation. However, other factors may also play a role.

\section{Authors' details}

${ }^{1}$ Institute of Tropical Medicine, Antwerp, Belgium. ${ }^{2}$ Aristide Le Dantec Teaching Hospital, Dakar, Senegal. ${ }^{3}$ Leiden University Medical Center, Leiden, the Netherlands.

Published: 17 September 2015

doi:10.1186/2049-3258-73-S1-P26

Cite this article as: Meurs et al:: Factors driving co-occurrence of

Schistosoma mansoni and S. haematobium at the micro-geographical level. Archives of Public Health 2015 73(Suppl 1):P26.

${ }^{1}$ Institute of Tropical Medicine, Antwerp, Belgium

Full list of author information is available at the end of the article 